\title{
The biomarkers of immune dysregulation and inflammation response in Parkinson disease
}

\author{
Li Chen ${ }^{1}$, Mingshu Mo ${ }^{1}$, Guangning Li ${ }^{7}$, Luan Cen², Lei Wei ${ }^{1,3}$, Yousheng Xiao ${ }^{2}$, Xiang Chen ${ }^{1}$, Shaomin Li ${ }^{4^{*}}$, \\ Xinling Yang ${ }^{5^{*}}$, Shaogang Qu ${ }^{6^{*}}$ and Pingyi $\mathrm{Xu}^{1,2^{*}}$
}

\begin{abstract}
Parkinson's disease (PD) is referring to the multi-systemic a-synucleinopathy with Lewy bodies deposited in midbrain. In ageing, the environmental and genetic factors work together and overactive major histocompatibility complex pathway to regulate immune reactions in central nerve system which resulting in neural degeneration, especially in dopaminergic neurons. As a series of biomarkers, the human leukocyte antigen genes with its related proteomics play cortical roles on the antigen presentation of major histocompatibility complex molecules to stimulate the differentiation of $\mathrm{T}$ lymphocytes and i-proteasome activities under their immune response to the PD-related environmental alteration and genetic variation. Furthermore, dopaminergic drugs change the biological characteristic of T lymphatic cells, affect the a-synuclein presentation pathway, and inhibit T lymphatic cells to release cytotoxicity in PD development. Taking together, the serum inflammatory factors and blood T cells are involved in the immune dysregulation of PD and inspected as the potential clinic biomarkers for PD prediction.
\end{abstract}

Keywords: Parkinson's disease, a-synucleinopathy, Inflammation, Biomarkers

Abbreviations: AD, Alzheimer's disease; APP, Amyloid precursor protein; CNS, Central neural system; CoREST, RE1-silencing transcription factor co-repressor 1; CSF, Cerebrospinal fluid; CTL, Cytotoxic T lymphocytes; DA, Dopaminergic; GBA, Glucosidase beta acid; HLA, Human leukocyte antigen; JNK-STAT, Janus kinase/signal transducers and activators of transcription; LMP2, Large multifunctional protease 2; LMP7, Large multifunctional protease 7; LRRK2, Leucine-rich repeat kinase 2; MDS, Movement Disorder Association; MECL-1, Multicatalytic endopeptidase complex-like-1; MHC, Major histocompatibility complex molecules; miRNAs, microRNAs; NK, Natural killer; NURR1, Nuclear receptor related 1 protein; PD, Parkinson's disease; PSMB, Proteasome subunit beta; SN, Substantia nigra; SNCA, Synuclein alpha; TAP, Antigen processing; UPDRS, Unified Parkinson's Disease Rating Scale; UPS, Ubiquitin proteasome system

\footnotetext{
*Correspondence: shaomin_li@hms.harvard.edu; Poplar862@sohu.com; sgq9528@163.com; pingyixu@sina.com

${ }^{4}$ Ann Romney Center for Neurologic Disease, Brigham and Women's Hospital, Harvard Medical School, Boston, MA 02115, USA

${ }^{5}$ Department of Neurology, The Third Affiliated Hospital of Xinjiang Medical University, Urumqi 830011, China

${ }^{6}$ Department of Blood Transfusion, The Fifth Affiliated Hospital, Southern Medical University, Guangzhou, Guangdong 510900, China

${ }^{1}$ Department of Neurology, The First Affiliated Hospital of Guangzhou Medical University, Guangdong 510120, China

Full list of author information is available at the end of the article
} 


\section{Background}

The raised incidence of Parkinson's disease (PD) becomes a serious issue in an aged society [1]. It is known that PD patients in Asia is nearby $11.3 \%$ of movement disorders, slightly lower than that in north America which is up to $13.6 \%$ and $16.6 \%$ of Europe [1]. Based on the characteristic protein conformation and function, central nervous degenerative diseases involved in movement disorders can be clinically classed as $\alpha$-synucleinopathy, Tauopathy and TDP-43 proteinopathy in which PD belongs to $\alpha$-synucleinopathy [2]. According to the latest clinic diagnosis criteria of MDS (Movement Disorder Association 2015), the diagnosis of PD need to meet the following criteria: rest tremor and bradykinesia in limbs, clinic symptoms of PD are effectively improved by L-dopa, disease duration is usually accompanied by non-motor symptoms especially in early stage, and discrimination from other neural disease [3]. The various types of $\alpha$-synuclein manifest mainly as monomers, oligomers, ribbons and fibrils, and asynuclein diffuses through blood brain barrier, spread around whole nervous system, even invade other tissues or organs such as nasal mucosa, skin, kidney, liver, heart, intestinal tract and salivary glands $[4,5]$. As well-known, aging is confirmed to be an important risk to PD [6]. Other risk factors include harmful environment factors and deficient genetic factors [6]. It's worth noting that environment factors such as MPTP, LPS, rotenone or other organic chemicals not only impair dopaminergic (DA) neurons directly and render the epigenetic variation of DNA methylation, but also activate the secondary inflammation/immunity reaction and significantly increase the PD morbidity $[7,8]$.

\section{Immunologic dysregulation in PD}

Recently immunologic systems were found in central neural system (CNS) which involved with PD [9]. The classic concepts pointed out that central immune system is not directly connected with extracranial system. However, latest reports demonstrated that lymphatic ducts hind in the meninge and link to deep cervicalvenous system in which abundant T, B lymphocytes and dendritic cells are response to environment and genetic variation [10]. According to recent dynamic experiments, the lymphatic system plays an important role in the drainage of spinal fluid to lymphatic duct oriented into deep cervical venous system [10]. This discovery revealed that there is a close linkage between the meninge lymphatic system and $\alpha$-synuclein transmission. The brain autopsy from PD patients and PD animal models showed that abnormal accumulation of microglia were activated in brain tissues, especially in nigrostriatal area [11]. Under the abnormal inflammation condition, macrophages pathologically diffuse from blood vessels to $\mathrm{CNS}$ and transform into microglias contributed to the pathological development of intracranial neural diseases [12]. Meanwhile the activated $\mathrm{T}$ lymphocytes were demonstrated to move out from central lymph ducts to attack central neurons, neural myelination or fibers, which resulting in multiple neuropathy as multiple sclerosis like symptoms [13]. In 6-hydroxydopamine-PD rats, there were abundant $\mathrm{CD}^{3+}, \mathrm{CD}^{4+}, \mathrm{CD}^{8+} \mathrm{T}$ lymph cells migrated from blood vessels into substantia nigra (SN) to attack DA neurons $[13,14]$. Therefore, it is reasonable to suppose these lymph cells could be the potential biomarkers for the evaluation of PD progression [14].

\section{Immunoproteasome system in PD}

The $\alpha$-synuclein aggregation is a critical ignition role for the immune disorder in PD [15]. In aging process, the progressive dysfunction of $\mathrm{T}, \mathrm{B}$ lymph cells and macrophages were related with immune elimination which gradually gave rise to $\alpha$-synuclein aggregation and neurons degeneration $[16,17]$. Furthermore, due to the decreased activity of proteasome, its components as $\beta 1, \beta 2$ and $\beta 5$ subunits which compose of $20 \mathrm{~S}$ proteasome will be replaced by homogenous $\beta 1 \mathrm{i}$ (large multifunctional protease 2, LMP2), $\beta 2 \mathrm{i}$ (multi catalytic endopeptidase complex-like-1, MECL-1) and 35 i (large multifunctional protease 7, LMP7) subunits and develop a new immunoproteasome system named as i-proteasome [18]. The crystal structure showed that i-proteasomes have more enzyme domains, stronger enzymatic activity, and more effective capacity to degrade the $\alpha$-synuclein proteins. Particularly, i-proteasome presents the degraded peptides as antigens to special T cells [19]. These antigens combined with major histocompatibility complex molecules (MHC) to ignite another pattern of protein clearance or abnormal neural inflammation [19]. It was reported that i-proteasome was up-regulated in senile hippocampus which involves in the clearance of accumulated amyloid precursor protein (APP) in Alzheimer's disease (AD) [20]. In epilepsy, the iproteasomes activated by oxidative damaged proteins can maintain the stability of protein metabolism in brain [21]. There are seven i-proteasome cleavage sites in the amino acid sequence of $\alpha$-synuclein (sequence No.100, $8,10,55,125,45$ and 18, respectively) [22]. Among them, No. 10 and 45 amino acid locate at $\mathrm{N}$ terminal named as KTKEGV sequence [22]. This KTKEGV sequence is vital to keep the metabolic stability of $\alpha$ synuclein [22]. The i-proteasomes mainly guide the MHC molecules to mediate inflammation/immunity reaction, however we did not completely understand the detail about i-proteasomes participating in PD pathogenesis. It is known that after the $\alpha$-synuclein degraded into 5-15 amino acid residues by ubiquitin proteasome system (UPS), the short antigenic peptides will be transported into endoplasmic reticulum system by the 
transporter associated with antigen processing (TAP) which brought the antigenic peptides to cell surfaces and the complex will be recognized by the MHC-I and MHC-II receptors. Then MHC presentation activates $\mathrm{CD}^{4+} \mathrm{T}$ cells and $\mathrm{CD}^{8+}$ cytotoxic $\mathrm{T}$ lymphocytes $(\mathrm{CTL})$ to take part in the immune regulation in CNS, respectively [23]. Human MHC molecules are also called human leukocyte antigen (HLA). DA neurons express multiple HLA molecules and present the digested peptides on the surface recognized by $\mathrm{CD}^{8+} \mathrm{CTL}$ cells [23]. At the same time, the activated microglias secrete TNF- $\alpha$, IL- $1 \beta$ and IL-6 inflammatory factors to attack DA neurons, or present antigens to $\mathrm{CD}^{4+} \mathrm{T}$ cells by MHC-II pathway $[24,25]$. Abundant MHC-II positive microglia and $\mathrm{CD}^{4}$ ${ }^{+}, \mathrm{CD}^{8+} \mathrm{T}$ cells were found in the SN of PD patients, and the microglia activity was confirmed to be related to the degeneration severity of DA neurons as well as PD progression [17]. Our data found that natural killer (NK) cells increased and $\mathrm{CD}^{3+} \mathrm{T}, \mathrm{CD}^{3+} \mathrm{CD}^{4+} \mathrm{T}$ cells, Th1 cells decreased in the peripheral blood from PD patients [26]. These immune reactions will result in the increased secretion of TNF- $\alpha$, IL-2, IL-6, IL-10, IL- $1 \beta$ and IFN- $\gamma$ in cerebrospinal fluid (CSF) or blood, and accompanied with more than ten kinds of high sensitive and specific antibodies increased, such as elongation factor 1-alpha1 and poly (A) binding protein cytoplasmic- $3[25,27]$. The surface antigens in monocytes were up-regulated and correlated with the Unified Parkinson's Disease Rating Scale (UPDRS) III scores of PD patients, indicating the importance of central and peripheral immunity reaction occurred in PD [28]. Recent studies showed that HLA-I in DA neurons and HLA-II in activated microglia both participate the antigen presentation and $\alpha$-synuclein degradation $[24,29]$. When proteasome function is suppressed by intracellular $\alpha$-synuclein accumulation, iproteasome will be activated as compensatory to promote $\alpha$-synuclein degradation [30]. The degraded peptides will be presented to $\mathrm{CD}^{8+} \mathrm{T}$ cells by MHC-I molecules, and the $\mathrm{CD}^{8+} \mathrm{T}$ cells release pre-inflammatory factors and activate Wnt/Nurr1 and Nuclear factor-kB signal pathways to ignite the secondary inflammation reaction [30,31]. Meanwhile, extracellular $\alpha$-synuclein integrates with HLA-DR molecules in microglia, mediate the activation of $\mathrm{CD}^{4+} \mathrm{T}$ cells by $\mathrm{MCH}-\mathrm{II}$ antigen presentation pathway and $\mathrm{T}$ lymphocytes to participate the immune reaction in CNS $[15$, $25]$. If the proteasomes function are impaired in DA neurons, $\alpha$-synuclein accumulation could activate the $\mathrm{i}$ proteasomeas as compensatory to provide much more MHC-I and MHC-II molecules to $\mathrm{CD}^{8+} \mathrm{T}$ and $\mathrm{CD}^{4+} \mathrm{T}$ cells showing stronger immune response [29, 32]. Under the condition of HLA gene mutation, the genetic risks may profoundly affect the $\alpha$-synuclein degradation, result in the immune dysregulation in CNS and finally accelerate the DA neurons apoptosis.

\section{Immunological genes involved in PD}

It is well known that genetic factors has high risk for PD. Much data revealed that the variation of HLA genes severely accelerate PD development [33]. Total 224 genes in HLA region are located at I, II and III region of HLA genomics, respectively [33]. Excepted the rarely expressed genes at HLA-I region, 128 genes from II and III of HLA region were found the contribution to immune regulation in CNS [33]. For example, HLA-DR at HLA II region is composed of DRA and DRB genes which encoding $\alpha$ and $\beta$ chain respectively [33]. The $\alpha$ and $\beta$ chains are assembled into HLA-DR molecules contributed to the immunologic response in neuron degeneration [33]. It's worth noting that HLA genes have high polymorphism among different ethnic and geographical population [34]. For example, HLA-C*0304, HLA-DRB1*0404, HLA-DRA and HLA-DQB1 were closely related to PD in European population, but no reports in Japanese [33, 34]. Our investigation firstly showed that HLA-DRB1*0301 is a suspect locus to PD in Chinese Han population $(\mathrm{OR}=2.048,95 \% \mathrm{CI}: 1.455,2.884)$, but no meaningful polymorphism found in Chinese Uyghur population [34]. It is known that proteasome subunit beta (PSMB) genes locate at HLA-II region and adjacent to HLA-DR genes [33]. PSMB genes encodes $\beta$ chain to help the $\alpha$-synuclein degradation after the proteasome constitution [35]. It was speculated that PSMB may cooperate with HLA-DR to participate the immune regulation and intrinsic antigen presentation [36]. Our investigation has confirmed that female carrying rs17587 G/G at PSMB9 will significantly increase PD risk $(\mathrm{OR}=1.85195 \% \mathrm{CI}$ : 1.240). The rs 17587 locates at the code region of exon 60 at PSMB9 [33]. Its variation of nucleotide $\mathrm{G}$ to A may lead to the amino acid of arginine replaced with histidine [37]. The G/G genotype of rs17587 at PSMB9 disrupts the peptide cleavage site of proteasome resulting in the impaired activity of proteasome [37]. Other important genes are reported to involve in immunological regulation of $\mathrm{PD}$, included HLA, GBA (Glucosidase Beta Acid), SNCA (Synuclein alpha), LRRK2 (Leucine-rich repeat kinase 2) and NURR1 (Nuclear receptor related 1 protein) [33, 38]. The GBA protein is liable to regulate the abnormal activation of microglia in basal ganglia [39], and the LRRK2 protein may affect the inflammatory reaction medicated by Toll-like receptor 4 [40]. Nurr1, a new anti-inflammatory factor, regulate the nuclear factor-kB-p65 signaling pathway in microglia and astrocytes through Nurr1/CoREST (RE1-silencing transcription factor co-repressor 1) complex [41]. Generally, variations in these PD-related genes may promote the antigen presentation and the activation of $\mathrm{T}$ and $\mathrm{B}$ lymphocytes, strengthen the sensitivity of DA neurons to environmental risk, and down-regulate the catecholamine metabolism conducted by MHC pathway [34]. 
In other words, it was suggested these genetic factors, serum immune/inflammatory molecules and blood lymphocytes may be the potential clinic biomarkers for PD prediction.

\section{Dopamine receptors in blood lymphocytes}

Dopamine receptors were found in the blood white cells of human kind [42]. These receptors are moderately expressed in neutrophil granulocyte or eosinophil granulocyte, but highly expressed in B lymphocytes and NK cells [42]. There are five kinds of dopamine receptors in $\mathrm{CD}^{3+} \mathrm{CD}^{4+} \mathrm{T}$ lymphocytes with different expression level [42]. For example, the D1 receptor expression is significantly higher than D2, and D5 receptor expression is higher than $\mathrm{D} 1$ in $\mathrm{T}$ lymphocytes and memory $\mathrm{T}$ lymphocytes [42]. The D2 receptor showed no significant different expression in blood cells from PD patient $[42,43]$. But the D3 receptor expression declines dramatically in peripheral lymphocytes and is associated with PD severity [42]. The proportion of CD95/CD3 increases significantly in the lymphocytes from PD patients, but decreases significantly after L-dopa treatment [44]. The activation of dopamine receptors by different activators can reduce the release of TNF- $\alpha$, IL- 6 and other cytokines from microglia which may help to explain their neuro-protection $[45,46]$.

\section{MicroRNA signatures in PD}

Recent studies demonstrated that some PD-related genes is modulated by $18 \sim 25 \mathrm{nt}$ microRNAs (miRNAs) [47] at post-transcriptional level. Matching with target mRNA 3' transcription terminals, miRNAs guide Dicer enzyme to degrade the mRNA targets or restrain their translation [48]. There are multiple mechanisms of miRNAs regulation on PD pathogenesis. For example, miR-7 and miR-153 modulate the mRNA level of $\alpha$ synuclein [49], LRRK2 contributes to the regulation of E2F1/DP expression which is affected by miR-7 and miR-184 [50]. Recent studies mainly focused on the microglia activation and macrophage infiltration in brain. It becomes a hot spot about the mechanism of miRNAs modulating the related inflammatory disorders in CNS. Based on function classification, some microRNAs are classified as pro-inflammatory molecules, such as miR-155, miR-125 and miR-101, and some are anti-inflammatory molecules, such as miR-146, miR-21 and miR-124 [51]. It was reported that miR-146 modulates the microglia activation in CNS regulated by NF-KB and JNK-STAT (Janus kinase/signal transducers and activators of transcription) pathways, miR-124 retrains macrophage and microglia activation conducted by CCAAT-enhancer-binding proteins- $\alpha-P U .1$ complex $[52,53]$. All the miRNAs are involved in the inflammation/immunity system on PD pathogenesis [54].
Furthermore, the decreased level of miR-141, miR214、miR-146b-5p and miR-193a-3p was found closely associated with the early stage of PD [55]. In our study, there were 644 abnormal miRNAs detected in A53T-PD transgenic mice using high-throughput screening. Among them, 72 miRNAs were confirmed to involve in the immune response in SN. The level of 42 miRNAs has changed significantly in midbrain, such as miR-146b-5 decreases to 0.45 fold, whereas miR-124$5 \mathrm{p}$ up to 0.44 fold. Several miRNAs were confirmed to be stable in blood, urine and CSF, so called circulating miRNAs [56]. We also screened out 10 circulating miRNAs in CSF samples from 40 Chinese PD patients for disease's inflammatory evaluation [57]. Among them, miR-200a-3p and miR-542-5p were supposed to be closely related to $\alpha$-synuclein aggregation, whereas miR-342-5p was proved to participate in modulating inflammatory reaction [57]. These circulating miRNAs are ideal biomarkers for the evaluation of CNS diseases.

\section{Conclusions}

In summary, the interaction of aging, environment risk and genetic factors results in the accumulation of $\alpha$-synuclein in midbrain which ignites the special inflammatory /immune response medicated by i-proteasome. In the inflammatory/immune procession, MHC signal pathway accompanied with microglia activation is vital to DA neurons apoptosis. Taking together, $\alpha$-synuclein accumulation and abnormal $\mathrm{MHC}$ antigenic presentation cause inflammation/immune response in CNS and provide specific biomarkers for the prediction of DA neurons degeneration and assessment of PD risk as well as PD development (Table 1).

Table 1 The biomarkers of immune dysregulation and inflammation response in PD

\begin{tabular}{|c|c|}
\hline $\begin{array}{l}\text { Immunological } \\
\text { genes }\end{array}$ & $\begin{array}{l}\text { PSMB9-rs17587, HLA-DRA, HLA-DRB1*0404, } \\
\text { HLA-DRB1*0301, HLA-C*0304, HLA-DQB1, etc. }\end{array}$ \\
\hline PD related genes & $\begin{array}{l}\text { PARK1, PARK2, PINK1, DJ-1, ATP13a2, GBA, } \\
\text { RAB7L1-NUCKS1, STK39, BST1, FAM47E-SCARB2, } \\
\text { SNCA, HLA, GPNMB, FGF20, LRRK2, GCH1, } \\
\text { MAPT, SREBF1, DDRGK1, NURR1, etc. }\end{array}$ \\
\hline Proteins in CSF & a-synuclein, A $\beta 42$, Tau, Nurr1, etc. \\
\hline Inflammation factors & TNF-a, IL-2, IL-6, IL-10, IL-1 $\beta$, IFN- $\gamma$, etc. \\
\hline Antibodies & $\begin{array}{l}\text { ICAM4, PTCD2, FRMD8, CTLA-4/FC, MYOT, HSH2D, } \\
\text { FN1, TRIM21, Elongation factor 1-alpha 1, } \\
\text { PABPC3, etc. }\end{array}$ \\
\hline Blood lymphocytes & $\begin{array}{l}\mathrm{CD}^{3+}, \mathrm{CD}^{4+}, \mathrm{CD}^{8+}, \mathrm{CD}^{31+} \mathrm{a} 4 \beta^{7+} \mathrm{CD}^{4+}, \mathrm{CD} 95 / \mathrm{CD} 3, \\
\text { NK cells, etc. }\end{array}$ \\
\hline PD related miRNAs & $\begin{array}{l}\text { miR-7, miR-153, miR-155, miR-125, miR-101, } \\
\text { miR-146, miR-21, miR-124, miR-141, miR-214, } \\
\text { miR-146b-5p, miR-193a-3p, miR-200a-3p, } \\
\text { miR-542-5p, miR-342-5p, etc. }\end{array}$ \\
\hline
\end{tabular}




\section{Acknowledgements}

Not applicable

\section{Funding}

This review was supported by research grants from the State Key Development Program for Basic Research of China (2011CB510000), the National Natural Science Foundation of China $(81271428,81471292$, U1503222 and 81430021) the keypoint Science Foundation of Guangdong of China (2015A030311021), a grant supported by technology project of Guangzhou (201604020152) and a grant supported by assisting research project of science and technology for Xinjiang (201591160). All founding were used for the design, collection, analysis and interpretation of data and in writing in the manuscript

\section{Availability of data and materials}

Not relevant.

\section{Authors' contributions}

All authors read and approved the final manuscript. LC and GL summarized the background. MM summarized the miRNA and i-proteasomes system part. $L C, L W, X C$ and $Y X$ summarized the inflammatory cells part. LC, MM and PX conceived, designed, and performed the paper. SL, XY and SQ revised the paper.

\section{Competing interests}

The authors declare they have no competing interest.

\section{Consent for publication}

Not relevant.

\section{Ethics approval and consent to participate}

Not relevant.

\section{Author details}

'Department of Neurology, The First Affiliated Hospital of Guangzhou Medical University, Guangdong 510120, China. ²Department of Neurology, The First Affiliated Hospital of Sun Yat-sen University, Guangdong 510080, China. ${ }^{3}$ Department of Neurology, The Third Affiliated Hospital of Sun Yat-sen University, Guangdong 510082, China. ${ }^{4}$ Ann Romney Center for Neurologic Disease, Brigham and Women's Hospital, Harvard Medical School, Boston, MA 02115, USA. ${ }^{5}$ Department of Neurology, The Third Affiliated Hospital of Xinjiang Medical University, Urumqi 830011, China. ${ }^{6}$ Department of Blood Transfusion, The Fifth Affiliated Hospital, Southern Medical University, Guangzhou, Guangdong 510900, China. ${ }^{7}$ Department of Neurology, The Affiliated Huadu Hospital of Southern Medical University, Guangzhou 510800, China.

Received: 18 March 2016 Accepted: 15 August 2016 Published online: 26 August 2016

\section{References}

1. Pringsheim T, Jette N, Frolkis A, Steeves TD. The prevalence of Parkinson's disease: A systematic review and meta-analysis. Mov Disord. 2014;29:1583-90.

2. Wakabayashi K, Tanji K, Mori F. [Pathology of basal ganglia in neurodegenerative diseases]. Brain and nerve $=$. Shinkei Kenkyu No Shinpo. 2009:61:429-39.

3. Postuma RB, et al. MDS clinical diagnostic criteria for Parkinson's disease Mov Disord. 2015:30:1591-601.

4. Peelaerts W. et al. Synuclein strains cause distinct synucleinopathies after local and systemic administration. Nature. 2015;522:340-344.

5. Tyson T, Steiner JA, Brundin P. Sorting Out Release, Uptake and Processing of Alpha-Synuclein During Prion-Like Spread of Pathology. J Neurochem. 2015; [Epub ahead of print].

6. Liu WM, et al. Time trends in the prevalence and incidence of Parkinson's disease in Taiwan: A nationwide, population-based study. J Fr Med Assoc. 2015;115:531-8.

7. Tsuboi Y. Environmental-genetic interactions in the pathogenesis of Parkinson's disease. Exp Neurobiol. 2012;21:123-8.

8. Trinh J, Guella I, Farrer MJ. Disease penetrance of late-onset parkinsonism: A meta-analysis. JAMA Neurol. 2014;71:1535-9.
9. Mosley RL, Hutter-Saunders JA, Stone DK, Gendelman HE. Inflammation and adaptive immunity in Parkinson's disease. Cold Spring Harb Perspect Med. 2012;2:a009381

10. Louveau A, et al. Structural and functional features of central nervous system lymphatic vessels. Nature. 2015;533:278.

11. Zhang P, Lokuta KM, Turner DE, Liu B. Synergistic dopaminergic neurotoxicity of manganese and lipopolysaccharide: differential involvement of microglia and astroglia. J Neurochem. 2010;112:434-43.

12. Wang $G$, et al. Microglia/macrophage polarization dynamics in white matter after traumatic brain injury. J Cereb Blood Flow Metab. 2013:33:1864-74

13. Odoardi $F$, et al. T cells become licensed in the lung to enter the central nervous system. Nature. 2012;488:675-9.

14. Wheeler CJ, et al. T-Lymphocyte Deficiency Exacerbates Behavioral Deficits in the 6-OHDA Unilateral Lesion Rat Model for Parkinson's Disease. J Neurol Neurophysiol. 2014;5:209.

15. Stuendl A, et al. Induction of a-synuclein aggregate formation by CSF exosomes from patients with Parkinson's disease and dementia with Lewy bodies. Brain. 2016:139:481-94.

16. Cabezas, R. et al. Astrocytic modulation of blood brain barrier: perspectives on Parkinson's disease. Front Cell Neurosci. (2014); 8.

17. Sanchez-Guajardo V, Barnum CJ, Tansey MG, Romero-Ramos M Neuroimmunological processes in Parkinson's disease and their relation to a-synuclein: microglia as the referee between neuronal processes and peripheral immunity. ASN Neuro. 2013;5:AN20120066.

18. Krüger $E$, Kloetzel P-M. Immunoproteasomes at the interface of innate and adaptive immune responses: two faces of one enzyme. Curr Opin Immunol. 2012:24:77-83.

19. Ferrington DA, Gregerson DS. Immunoproteasomes: structure, function, and antigen presentation. Prog Mol Biol Transl Sci. 2012;109:75.

20. Orre $M$, et al. Reactive glia show increased immunoproteasome activity in Alzheimer's disease. Brain. 2013;136:1415-31.

21. Mishto $M$, et al. The immunoproteasome $\beta 5$ i subunit is a key contributor to ictogenesis in a rat model of chronic epilepsy. Brain Behav Immun. 2015:49:188-96.

22. Dettmer U, Newman AJ, von Saucken VE, Bartels T, Selkoe D. KTKEGV repeat motifs are key mediators of normal a-synuclein tetramerization: Their mutation causes excess monomers and neurotoxicity. Proc Natl Acad Sci. 2015;112:9596-601.

23. Neefjes J, Jongsma ML, Paul P, Bakke O. Towards a systems understanding of MHC class I and MHC class II antigen presentation. Nat Rev Immunol. 2011;11:823-36

24. Cebrián C, et al. MHC-I expression renders catecholaminergic neurons susceptible to T-cell-mediated degeneration. Nat Commun. 2014;16:3633.

25. More SV, Kumar H, Kim IS, Song S-Y, Choi D-K. Cellular and molecular mediators of neuroinflammation in the pathogenesis of Parkinson's disease. Mediators of inflamm. 2013:952375

26. Zhang S, et al. Clinical analysis of subpopulation of peripheral T and B lymphocytes in Chinese Parkinson's disease patients. Zhonghua Yi Xue Za Zhi. 2014:94(47):3726-30.

27. Santiago JA, Potashkin JA. Current Challenges Towards the Development of a Blood Test for Parkinson's Disease. Diagnostics. 2014:4:153-64.

28. Saunders JAH, et al. CD4+ regulatory and effector/memory T cell subsets profile motor dysfunction in Parkinson's disease. J Neuroimmune Pharmacol. 2012;7:927-38.

29. Starossom SC, et al. Galectin-1 deactivates classically activated microglia and protects from inflammation-induced neurodegeneration. Immunity. 2012;37:249-63.

30. Zhang L-M, et al. Dopamine Agonists Exert Nurr1-inducing Effect in Peripheral Blood Mononuclear Cells of Patients with Parkinson's Disease. Chin Med J. 2015:128:1755.

31. Wei $L$, et al. Activation of Wnt/ß-catenin pathway by exogenous Wnt protects SH-SY5Y cells against 6-hydroxydopamine toxicity. J Mol Neurosci. 2013;49:105-15.

32. Heneka MT, Kummer MP, Latz E. Innate immune activation in neurodegenerative disease. Nat Rev Immunol. 2014;14:463-77.

33. Mo M, et al. Association Analysis of Proteasome Subunits and Transporte Associated with Antigen Processing on Chinese Patients with Parkinson's Disease. Chin Med J. 2016:129:1053.

34. Sun C, et al. HLA-DRB1 alleles are associated with the susceptibility to sporadic Parkinson's disease in Chinese Han population. PLoS One. 2012; 7(11):e48594. 
35. Allcock RJN. The major histocompatibility complex: a paradigm for studies of the human genome. Immunogenetics: Methods and Applications in Clinical Practice. 2012:1-7.

36. Rhodes DA. Human Leukocyte Antigen (HLA) System and Human Disorders. eLS. 2005.

37. Camarena Á, et al. PSMB8 (LMP7) but not PSMB9 (LMP2) gene polymorphisms are associated to pigeon breeder's hypersensitivity pneumonitis. Respir Med. 2010;104:889-94.

38. Nalls MA, et al. Large-scale meta-analysis of genome-wide association data identifies six new risk loci for Parkinson's disease. Nat genet. 2014;46:989-93.

39. Barkhuizen M, Anderson DG, Grobler AF. Advances in GBA-associated Parkinson's disease-pathology, presentation and therapies. Neurochem Int. 2015;93:6-25.

40. Hsu CH, et al. MKK6 binds and regulates expression of Parkinson's diseaserelated protein LRRK2. J Neurochem. 2010;112:1593-604.

41. Smith GA, et al. A Nurr1 Agonist Causes Neuroprotection in a Parkinson's Disease Lesion Model Primed with the Toll-Like Receptor 3 dsRNA Inflammatory Stimulant Poly (I: C). PLoS One. 2015;10:e0121072.

42. González H, et al. Dopamine receptor D3 expressed on CD4+ T cells favors neurodegeneration of dopaminergic neurons during Parkinson's disease. J Immunol. 2013;190:5048-56.

43. Stevens $\mathrm{CH}$, et al. Reduced Thelper and B lymphocytes in Parkinson's disease. J Neuroimmunol. 2012;252:95-9.

44. Hurny A, Michałowska-Wender G, Wender M. Impact of L-DOPA treatment of patients with Parkinson's disease on mononuclear subsets and phagocytosis in the peripheral blood. Folia Neuropathol. 2013;51:127-31.

45. Kumar A, Sharma N, Gupta A, Kalonia H, Mishra J. Neuroprotective potential of atorvastatin and simvastatin (HMG-CoA reductase inhibitors) against 6-hydroxydopamine (6-OHDA) induced Parkinson-like symptoms. Brain Res. 2012;1471:13-22

46. Sanchez-Guajardo V, Annibali A, Jensen PH, Romero-Ramos M. a-Synuclein Vaccination Prevents the Accumulation of Parkinson Disease-Like Pathologic Inclusions in Striatum in Association With Regulatory T Cell Recruitment in a Rat Model. J Neuropathol Exp Neurol. 2013;72:624-45.

47. Mouradian MM. MicroRNAs in Parkinson's disease. Neurobiol Dis. 2012;46:279-84.

48. Abe M, Bonini NM. MicroRNAs and neurodegeneration: role and impact Trends Cell Biol. 2013;23:30-6.

49. Alvarez-Erviti $L$, et al. Influence of microRNA deregulation on chaperonemediated autophagy and a-synuclein pathology in Parkinson's disease. Cell Death Dis. 2013;4:e545.

50. Chan SL, Angeles DC, Tan E-K. Targeting leucine-rich repeat kinase 2 in Parkinson's disease. Expert Opin Ther Targets. 2013;17:1471-82.

51. Ponomarev ED, Veremeyko T, Weiner HL. MicroRNAs are universal regulators of differentiation, activation, and polarization of microglia and macrophages in normal and diseased CNS. Glia. 2013:61:91-103.

52. Svahn AJ, Giacomotto J, Graeber MB, Rinkwitz S, Becker TS. miR-124 Contributes to the functional maturity of microglia. Dev Neurobiol. 2015;76:507-18.

53. Saba R, et al. MicroRNA 146a (miR-146a) is over-expressed during prion disease and modulates the innate immune response and the microglial activation state. PLoS One. 2012;7:e30832.

54. Wang $\mathrm{H}$, et al. MiR-124 Regulates Apoptosis and Autophagy Process in MPTP Model of Parkinson's Disease by Targeting to Bim. Brain Pathol. 2015; 26:167-76.

55. Dong $\mathrm{H}$, et al. A panel of four decreased serum microRNAs as a novel biomarker for early Parkinson's disease. Biomarkers. 2016;21:129-37.

56. Jeck WR, Sharpless NE. Detecting and characterizing circular RNAs. Nat Biotechnol. 2014;32:453-61.

57. Wei $Y$, et al. The microRNA-342-5p Fosters Inflammatory Macrophage Activation Through an Akt1-and microRNA-155-Dependent Pathway During Atherosclerosis. Circulation. 2013;127:1609-19.

\section{Submit your next manuscript to BioMed Central and we will help you at every step:}

- We accept pre-submission inquiries

- Our selector tool helps you to find the most relevant journal

- We provide round the clock customer support

- Convenient online submission

- Thorough peer review

- Inclusion in PubMed and all major indexing services

- Maximum visibility for your research

Submit your manuscript at www.biomedcentral.com/submit
Biomed Central 